\title{
19.
}

\section{Der allgemeine Satz über die Erzeugung aller algebraischer Curven durch Bewegung gerader Linien.}

(Von Herrn Professor Dr. H. Gra/smann, Oberlehrer der Mathematik zu Stettin.)

Vor längerer Zeit habe ich in dem ersten Theile meiner Ausdehnungslehre (\$. 145-148.) einen allgemeinen Satz mitgetheilt über die Erzeugung der Curven höherer Ordnungen, so wie der algebraischen Oberflächen, durch Bewegung gerader Linien oder Ebenen; und in diesem Journale (Band 31. und 36.) habe ich besondere Anwendungen desselben, besonders auf Curven dritter Ordnung gegeben. Die Bearbeitung des zweiten Theils jenes Werks, den ich jetzt unter der Feder habe, hat mich wieder auf den Gegenstand zurückgeführt, und ich bin dabei zu einer Reihe neuer Resultate gelangt, von denen ich diejenigen, welche sich an die in den erwähnten Abhandlungen dargestellte Analyse anschliefsen, den Lesern dieses Journals in einer Reihe von Aufsätzen mitzutheilen beabsichtige.

Der oben erwähnte Satz, dem ich hier eine wesentliche Ergänzung hinzufügen will, findet sich im 31. Bande dieses Journals in folgender Form ausgesprochen :

"Wenn die Lage eines beweglichen Punctes $x$ in der Ebene dadurch beschränkt ist, dafs ein Punct und eine Gerade, welche durch Constructionen vermittels des Lineals aus jenem Puncte $x$ und einer Reihe fester Puncte und Geraden hervorgehen, zusammenliegen sollen (d. h. der Punct in der Geraden liegen soll): so beschreibt der Punct $x$ ein algebraisches Punct-Gebilde, und zwar ein Punctgebilde nten Grades (eine Curve nter Ordnung), wenn bei den Constructionen der bewegliche Punct $n$ mal angewandt ist."

Den Beweis dieses Satzes, der eine Erweiterung des Pascalschen Satzes über das mystische Sechseck ist, habe ich in der Ausdehnungslehre aus den Crelle's Journal f. d. M. Bd. XLII. Heft 3. 26 
Principien dieser Wissenschaft, und im 31. Bande dieses Journals aus der gewöhnlichen Analyse hergeleitet, und habe diesem Beweise hier nichts hinzuzufügen. Um jedoch den Satz einer allgemeinen Behandlung algebraischer Curven zu Grunde legen zu können, bedarf derselbe noch einer Ergänzung; indem nämlich gezeigt werden mufs, dafs auch umgekehrt jede algebraische Curve auf die in dem Satze angegebene Weise erzeugt werden kann. Und Dies nachzuweisen ist der Hauptzweck des gegenwärtigen Aufsatzes.

Ist $f(x, y)=0$ die Gleichung einer algebraischen Curve, bezogen auf irgend ein Axenkreuz, also $f(x, y)$ eine ganze rationale Function von $x$ und $y$, so geht diese Function aus $x, y$ und den Constanten durch Addition und Multiplication hervor. Es kommt also nur darauf an, die Addition und Multiplication zweier Zahlgröfsen durch lineale Construction darzustellen. Unter linealer Construction verstehe ich nicht nur die Verbindung zweier endlich entfernter Puncte durch eine gerade Linie, sondern auch das Ziehen der Parallele, oder, anders ausgedrückt, die Verbindung eines endlich und eines unendlich entfernten Punctes durch eine Gerade; also überhaupt das Verbinden zweier Puncte durch eine Gerade. Um nun die Addition und Multiplication durch geometrische Constructionen darzustellen, kommt es darauf an, jede der zu verknüpfenden Zahlgröfsen durch geometrische Gröfsen zu ersetzen. Ich nehme zwei Coordinaten-Axen an, die sich in $c$ durchschneiden, und auf jeder derselben ein bestimmtes Stück als Maafs; es sei dies $c a$ auf der $x$ Axe (Fig. 1.), und $c b$ auf der $y$ Axe; wobei es ganz gleichgültig ist, ob diese beiden Maafse gleich lang sind, oder nicht. Durch das Maafs ca seien die Abscissen, durch das Maafs $c b$ die Ordinaten gemessen, und die Quotienten dieser Messungen seien eben $x$ und $y$. Der Endpunct der Abscisse sei $x^{\prime}$; von dem Endpuncte der Ordinate sei, um alle Gröfsen auf derselben Linie, der Abscissenaxe, zu haben, die Parallele mit ba gezogen, welche die Abscissen in $y^{\prime}$ schneide: dann ist

$$
x=\frac{c x^{\prime}}{c a}, \quad y=\frac{c y^{\prime}}{c a} \text {. }
$$

Auch ist klar, wie sowohl $x^{\prime}$ als $y^{\prime}$ aus dem die Curve construirenden Puncte, den wir $p$ nennen wollen, durch lineale Constructionen erfolgen (Fig. 1.). Ferner nehmen wir an, dafs auch jede Constante in der Function $f(x, y)$ durch einen Punct der Geraden $c a$ in der Art dargestellt sei, dafs die Entfernung dieses Puncts von dem Anfangspunct $c$, gemessen durch das Maafs $c a$, jener Constanten gleich sei. Auf diese Weise sind dann alle in der Function 
vorkommenden Gröfsen durch Puncte der Abscissenaxe dargestellt, und es kommt nur darauf an, auch die Summe und das Product zwei solcher Gröfsen auf gleiche Weise durch Puncte dieser Linie vermittels linearer Constructionen darzustellen. Es seien (Fig. 2.) zuerst $f$ und $g$ zwei solche Puncte und $h$ der gesuchte, also im ersten Falle

$$
\frac{c f}{c a}+\frac{c z}{c a}=\frac{c h}{c a} \quad \text { oder } \quad c f+c g=c h .
$$

Die einfachste lineale Construction des Puncts $h$ ist die, dafs man einen Punct $d$ aufserhalb der Geraden $c f$ zu Hülfe nimmt, von $f$ die Parallele mit $c d$, von $d$ die mil $c f$ zieht, und von dem Durchschnitt $e$ dieser beiden Linien die Parallele mit $d g$ zieht, welche die Gerade $c f$ in dem gesuchten Puncte $h$ schneidet. Für die Multiplication hat man

$$
\frac{c f}{c a} \cdot \frac{c g}{c a}=\frac{c h}{c a} \quad \text { oder } \quad c f \cdot c g=c h . c a .
$$

Aus der Proportion $c a: c f=c g: c h$ ergiebt sich dann die lineale Construction von h unmittelbar. Hieraus folgt also, dafs $f(x, y)$ aus dem die Curve construirenden Puncte $\boldsymbol{p}$ und constanten Puncten und Geraden sich lineal construiren läfst. Soll nun $f(x, y)$ gleich Null sein, so mufs der zu $f(x, y)$ gehörige Punct in $c$ fallen, also die Gerade, deren Durchschnitt mit ca den zu $f(x, y)$ gehörigen Punct liefert, mufs durch $c$ gehen: d. h. es findet die in dem Hauptsatze ausgesprochene Bedingung Statt, dafs ein Punct und eine Gerade, welche aus linealen Constructionen hervorgehen, zusammenliegen sollen; also ist die gegebene Curve durch die in dem Hauptsatz angegebene Construction erzeugbar. Q. d. e.

Um den Hauptsatz für die Anwendung hequemer zu machen, will ich den Begriff der offenen Figur und der Verkettung von geraden Linien einführen. Die offene Figur (S. dieses Journal Band 36.) besteht aus einer Reihe von Puncten und geraden Linien, in der Art, dafs auf jeden Punct eine durch ihn gehende Gerade, und auf jede Gerade ein in ihr liegender Punct folgt, bis endlich die Reihe entweder mit einem Puncte, oder mit einer Geraden schliefst; wie sie denn auch entweder mit einem Puncte oder mit einer Geraden beginnt. Punct und Gerade nenne ich zusammen Elemente; das Element, mit welchem jene Reihe beginnt, oder schliefst, nenne ich Anfangs-oder End-Elernent, beide zusammen Gränz-Elemente; alle Puncte oder Geraden jener Reihe, die 
nicht Gränz-Elemente sind, nenne ich Ecken oder Seiten der offenen Figur. Wenn man nun (Fig. 3.) ein Element $x$ zum Anfangs-Element mehrerer offenen Figuren macht, dann unter den so gewonnenen offenen Figuren zwei beliebige so zusammenschliefst, dafs sie ein gemeinschaftliches End-Element erhalten, welches zugleich Anfangs-Element einer neuen offenen Figur wird, und beliebig fortfährt, die jedesmal noch übrigen offenen Figuren auf die angegebene Weise paarweise zusammenzuschliefsen, so will ich die so hervorgehende Figur eine Verkettung gerader Linien nennen; das Element $x$ soll das Anfangs-Element dieser Verkettung, die sämmtlichen übrigen Gränz-Elemente der offenen Figuren sollen die Übergangs-Elemente der Verkettung heifsen, während ich die Ecken und Seiten der offenen Figuren zugleich als Ecken und Seiten der Verkettung setze. Wenn insbesondere zuletzt nur Eine offene Figur übrig bleibt, deren End-Element mit dem Anfangs-Element $x$ der Verkettung zusammenfällt, so nenne ich die Verkettung eine geschlossene; und zwar vom nten Grade, wenn von dem Anfangs-Element $(\boldsymbol{x}) \boldsymbol{n}$ offene Figuren ausgehen (die letzte mit eingerechnet, welche $x$ zum Elemente hat). Dann lautet der Satz wie folgt:

„Wenn sich eine Verkettung nten Grades lineal, d. h. so bewegt, dafs alle Seiten durch feste Puncte gehen und alle Ecken in festen Geraden bleiben, so beschreibt das Anfangs-Element der Verkettung ein Gebilde nten Grades."

Wendet man diesen Satz z. B. auf die Curven zweiter, dritter und vierter Ordnung an, so erhält man folgende abgeleiteten Sätze:

1. Wenn in einer geschlossenen Figur alle Ecken und Seiten, mit Ausnahme einer Ecke, sich lineal bewegen (d. h. die Ecken in festen Geraden, die Seiten um feste Puncte), so beschreibt diese lelzte Ecke einen Kegelschnitt.

2. Wenn drei offene Figuren, mit gemeinschaftlichen Gränz-Elementen, sich lineal bowegen (d. h. so, dafs die Ecken in festen Geraden bleiben, die Seiten durch feste Puncte gehen), so beschreibt jedes der Gränz-Elemente ein Gebilde dritten Grades (S. dieses Journal Band 36.).

3. Wenn fünf offene Figuren lich lineal bewegen, von denen vier alle dasselbe Anfangs-Element $(x)$ und paarweise dieselben End-Elemente $(y, z)$ haben, wăhrend die Gränz-Elemente der fünften mit diesen End-Elementen $(y, *)$ zusammenfallen, so beschreibt das Anfangs-Element $(x)$ ein Gebilde vierten Grades (S. Fig. 3.). 
Ich füge hier noch zwei Bemerkungen hinzu, welche zur Erläuterung und richtigen Anwendung des Hauptsatzes dienen werden. Zuerst ist es klar, dafs von den offenen Figuren einige zusammenfallen können, und zwar in der Art, dafs sie auch gleiche Gränz-Elemente haben. Dies Zusammenfallen wird sich dann in der Verkettung selbst nur dadurch zu erkennen geben, dafs ein folgendes Übergangs-Element mehr als drei Gränz-Elemente in sich vereinigt. Der Grad der Verkettung, und also auch des erzeugten Gebildes, wird sich auch in diesem Falle leicht angeben lassen. Es werde z. B. in (Fig. 3.) der Weg gesucht, den $g$ beschreibt, also $g$ als Anfangspunct der Verkettung gesetzt. Alsdann gehen von dem Übergangs -Element $x$, aufser den beiden offenen Figuren, die von $y$ direct nach $x$ gehen, noch zwei offene Figuren aus: mithin müssen jene ersteren beiden doppelt gerechnet werden, und es ist die Verkettung vom fünften Grade; $y$ beschreibt also eine Curve fünften Grades, und Dasselbe gilt von z. Es würde sich leicht nachweisen lassen, dafs in solchen Fällen jedesmal Curven mit Doppelpuncten erzeugt werden (Vergl. darüber die Abhandlung in dem 31. Bande Seite 20). Doch möge dieser Nachweis dem Leser überlassen bleiben.

Die zweite Bemerkung bezieht sich auf die Übergangsfälle, in welchen der Grad des Gebildes scheinbar niedriger wird. Dies kann auf zweifache Art geschehen, indem entweder die ganze Function $n$ ten Grades $f(x, y)$, welche, gleich Null gesetzt, die Curve bestimmt, in Factoren sich zerfällen läfst, von denen zwei oder mehrere einander gleich sind: oder wenn Coëfficienten Null werden und dadurch die Glieder höherer Grade wegfallen; ja es könnten alle Coëfficienten Null und dadurch die Curve ganz unbestimmt werden. In allen diesen Fällen wird man jedoch durch Variation der Constanten sogleich die Curve nter Ordnung wieder in Evidenz bringen können; und insofern wir also jene besondern Fälle nur als Übergangsfälle betrachten, in denen die allgemeinen Constanten gewisse besondere Werthe annehmen, werden wir auch diese Übergangsgebilde als Gebilde $n$ ten Grades setzen müssen. Nur unter dieser Voraussetzung hat der aufgestellte Satz seine vollkommen allgemeine Bedeutung; wie denn auch alle allgemeinen Sätze über algebraische Curven nur unter dieser Voraussetzung gelten. Schliefst man das unbestimmte Gebilde nten Grades aus und nimmt an, dafs die sämmtlichen Coëfficienten der Glieder der $m$ höchsten Grade verschwinden, so bleibt die Curve vom $(n-m)$ ten Grade; und um 
19. Grafsmann, über die Erzeugung algebraischer Curven.

sie als Curve nten Grades aufzufassen, hat man $m$ gerade Linien, welche ins Unendliche fallen, mit der Curve $(n-m)$ ter Ordnung zusammenzufassen. Obgleich das so eben Gesagte hinlänglich bekannt ist, so glaubte ich es doch hier noch einmal in Erinnerung bringen zu müssen, da die Art, wie wir aus einer Gleichung nten Grades die lineale Erzeugung der betreffenden Curve ableiteten, stets, wenn die Gleichung mehr als ein variables Glied enthält, auf ein Gebilde von höherem als dem nten Grade hinführt, welches sich aber in ein Gebilde nten Grades und in eine Reihe von geraden Linien die ins Unendliche fallen, zerfällen läfst. Ich denke, auf diese besonderen Verhältnisse in einer späteren Abhandlnng zurückzukommen.

Stettin, im Juli 1851. 